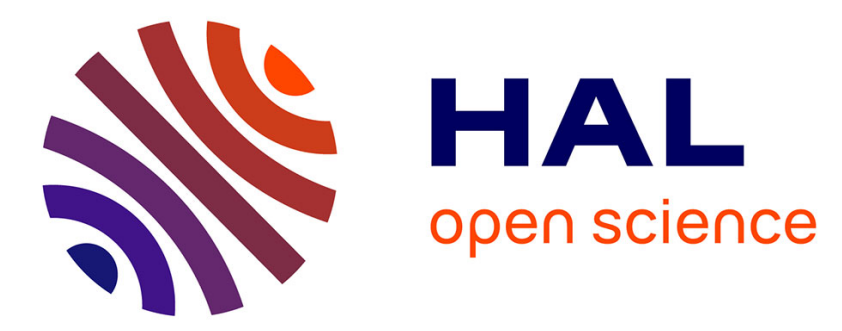

\title{
Periodic Area-of-Motion characterization for Bio-Medical applications
}

\author{
Élodie Puybareau, Hugues Talbot, Laurent Najman
}

\section{To cite this version:}

Élodie Puybareau, Hugues Talbot, Laurent Najman. Periodic Area-of-Motion characterization for Bio-Medical applications. ISBI 2017, Apr 2017, Melbourne, Australia. 10.1109/ISBI.2017.7950503 . hal-01467878

\section{HAL Id: hal-01467878 https://hal.science/hal-01467878}

Submitted on 14 Feb 2017

HAL is a multi-disciplinary open access archive for the deposit and dissemination of scientific research documents, whether they are published or not. The documents may come from teaching and research institutions in France or abroad, or from public or private research centers.
L'archive ouverte pluridisciplinaire HAL, est destinée au dépôt et à la diffusion de documents scientifiques de niveau recherche, publiés ou non, émanant des établissements d'enseignement et de recherche français ou étrangers, des laboratoires publics ou privés. 


\title{
Periodic Area-of-Motion characterization for Bio-Medical applications
}

\author{
Elodie Puybareau ${ }^{1,2}$, Hugues Talbot ${ }^{1}$, Laurent Najman ${ }^{1}$ \\ ${ }^{1}$ Université Paris-Est ESIEE, LIGM, UMR CNRS 8049, 2 Boulevard Blaise-Pascal, F-93162 Noisy-le-Grand. \\ ${ }^{2}$ EPITA Research and Development Laboratory (LRDE), 14-16, rue Voltaire, F-94276 Le Kremlin-Bicêtre
}

\begin{abstract}
Many bio-medical applications involve the analysis of sequences for motion characterization. In this article, we consider 2D+t sequences where a particular motion (e.g. a blood flow) is associated with a specific area of the 2D image (e.g. an artery) but multiple motions may exist simultaneously in the same sequences (e.g. there may be several blood vessels present, each with their specific flow). The characterization of this type of motion typically involves first finding the areas where motion is present, followed by an analysis of these motions: speed, regularity, frequency, etc. In this article, we propose a methodology called "area-of-motion characterization" suitable for simultaneously detecting and characterizing areas where motion is present in a sequence. We can then classify this motion into consistent areas using unsupervised learning and produce directly usable metrics for various applications. We illustrate this methodology for the analysis of cilia motion on ex-vivo human samples, and we apply and validate the same methodology for blood flow analysis in fish embryo.
\end{abstract}

Index Terms- Fourier analysis, classification, unsupervised learning, cilia, flow analysis.

\section{INTRODUCTION}

Sequence analysis, and in particular those that involve motion, is common in bio-medical applications [1]. In this article, our domain of application concerns areas-of-motion characterization in $2 \mathrm{D}+\mathrm{t}$ sequences. This means that we assume that in our sequences of interest, we can tessellate the 2D domain into consistent areas that may undergo various degrees of patterned geometry evolution, texture changes or grey-level intensity variation. We also assume that these areas-of-motion remain unchanged through the sequence. Note that this is a very different problem from object tracking, where an otherwise broadly unchanging, identifiable object might move through the sequence (e.g a fish swimming) in a non-repeating pattern.

In general terms, to characterize areas-of-motion in an image sequence, it is therefore necessary to find areas in the sequence of interest where some motion occurs. Since these areas are assumed fixed, but motion occurs in there repeatedly, it seems natural to assume that these motions are periodic or quasi periodic, i.e. they occur with significant, but not metronomic regularity. If we take as example the case of a sequence of an otherwise unmoving but beating heart in a transparent fish, we may find areas in the sequence that correspond to geometry changes (the moving contours of the heart) and others where only grey-level changes are visible (as the ventricles of heart fills and empties with blood). However we may discover that these separate changes are linked and consistent because they are spatially related, synchronized and periodic, and therefore belong to the same object.

This suggests using motion analysis descriptors to these areas that are as parameter free as possible and can distinguish areas of motion based on their combined response. Assuming quasi periodic motion, we propose to use multiscale Fourier domain analysis. Because we do not assume the availability of labelled or annotated data, we propose to use an unsupervised learning approach, so that areas of consistent motion can be detected by clustering methods.

Although sometime described in a different way, areaof-motion tessellation has been studied before: In [2], authors propose a joint segmentation and motion analysis in a Bayesian framework. In [3], periodic motion segmentation is investigated. In [4], a multiple level-line framework is used to delineate independent areas of motion. In [5], unsupervised spectral clustering is used to detect these areas over a few frames. In previous work [6, 7], we have used variance analysis on temporal gradient to propose area-of-motion identification. In [8], we used optical flow [9] for area of motion clustering. In this new work, we combine the strong prior knowledge of repeating motion with unsupervised clustering to propose a flexible and robust approach for area-of-motion detection. We also show how our proposed descriptors can be used for motion characterization in the detected areas, with applications to several bio-medical problems.

The remainder of the article is structured as follows: in section 2, we propose suitable motion analysis descriptors. In section 3 we describe our unsupervised learning framework. Section 4 shows our results on several applications. We conclude in section 5 . 
Fig. 1. Illustration of neighbors centroïds with their areas.

\section{PROPOSED MOTION DESCRIPTORS}

A statistical process that retains its defining characteristics (e.g. amplitude, density, frequency, etc) through time is called stationary in time. A process that has varying characteristics (i.e. parameters) depending on the location is called spatially variant. Areas of motion are simultaneously stationary in time and spatially variant. In this section, we propose descriptors that are suitable for these types of processes.

\subsection{Nearly stationary processes}

Periodic processes constitute a large subclass of stationaryin-time processes. The processes that we study are assumed to be nearly periodic (i.e, as much as biological processes can be), and we only expect slow changes in motion patterns during analysis, so they can be assimilated to nearly stationaryin-time, periodic processes. A standard tool to study these processes is the Fourier Transform (FT). Continuous wavelets like the Gabor-Morlet wavelets $[10,11]$ are designed for the frequency analysis of non-stationary processes, so they are not ideal here. Discrete wavelets (Haar, Daubechies, etc) are even less suitable since they are more associated with the notion of scale than with the notion of frequency. Our proposed approach consists of comparing Fourier spectra in small neighboring regions.

\subsection{Calculating the descriptors}

Let $I$ be a 2D+t sequence of spatial size $M \times N$ and consisting of $F$ frames indexed $\{1 \ldots F\}$. We distinguish the notion of a 2D location $l$ at coordinates $(i, j)$, which remains unchanged throughout the sequence, from that of a pixel $p$ of coordinates $(i, j, t)$, located in frame $t$. We denote $I(l)$ the 1D sequence of $F$ values, e.g. $I(l)=\{I(i, j, 1), \ldots, I(i, j, F)\}$.

Let $l, m$ be neighboring locations in the sequence. We consider a square window $\mathcal{R}_{l}$ (resp. $\left.\mathcal{R}_{m}\right)$ of width $(2 r+$ 1) pixels centered on these (see Fig 1 ). so each region $\mathcal{R}_{l}$ contains $(2 r+1)^{2}$ spectra.

We denote $s_{k}$ the power spectrum at location $k$ belonging to the window $\mathcal{R}_{l}$. The considered spectrum $\mathcal{S}_{l}$ associated this window is the average of all the $s_{k}$, i.e.:

$$
\begin{aligned}
s_{k} & =\mathcal{F}(I(k)) \\
\mathcal{S}_{l} & =\frac{1}{(2 r+1)^{2}} \sum_{k(i, j) \in \mathcal{R}_{l}}\left\|s_{k}\right\|,
\end{aligned}
$$

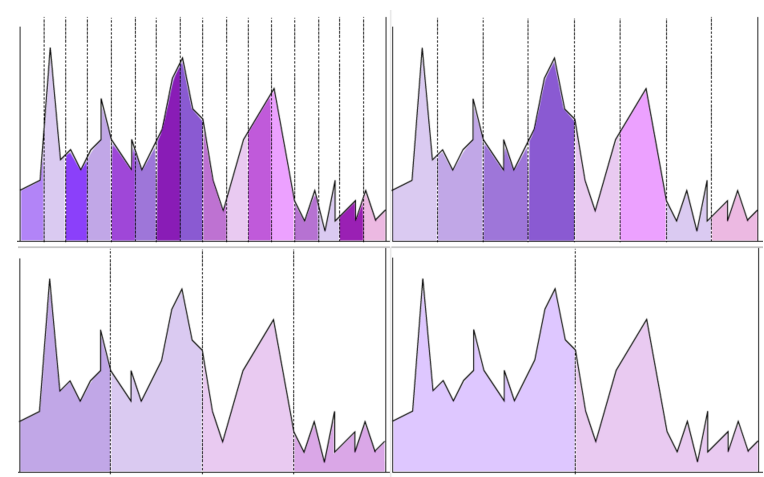

Fig. 2. Multiscale merging and averaging of the FFT spectrum for calculating the $V_{l}$

where $\mathcal{F}$ denotes the Fourier transform. Since we consider the power spectrum, only the components $[1, F / 2]$ of $\mathcal{S}_{l}$ are relevant since the components $[F / 2+1, F]$ are symmetrical.

We then compute $F-1$ descriptors $\left\{V_{l}[1] \ldots V_{l}[F-1]\right\}$ from $S_{l}[1, F / 2]$. They are obtained by considering the low pass Haar wavelet decomposition of the FFT spectrum, i.e. by recursively averaging and merging the vector $\mathcal{S}_{l}$ (see Fig. 2). In particular we have the properties

$$
\begin{aligned}
V_{l}\left[\frac{F}{2}+g-1\right] & =\mathcal{S}[g] \forall g \in\left[1, \frac{F}{2}\right] \\
V_{l}[h] & =V[2 h]+V[2 h+1] \quad \forall h \in\left[\frac{F}{2}, 1\right]
\end{aligned}
$$

There are indeed $\sum_{i=0}^{\log _{2}(F / 2)} 2^{i}=F-1$ such descriptors. In this way the resulting descriptors corresponds to a multiscale wavelet representation of the average power frequency spectrum of the region around the location of interest. It is inspired by but different from a multiscale spatial representation found in standard wavelets. We can also consider the high-pass coefficients $W_{l}$ defined in this way:

$$
W_{l}[h]=V[2 h]-V[2 h+1] \quad \forall h \in\left[\frac{F}{2}, 1\right],
$$

but we did not use them in this work as they are fairly noisy. In practice we do not need to consider all the wavelet coefficients, only those near the low-pass area of the spectrum (for example in the interval $\left[1, \frac{F}{8}\right]$ ), since most of the highfrequency part of the power spectrum is also noise. To further reduce noise we may also only keep some of the averaged descriptors, for instance only the first $\left\{V_{t}[1], \ldots, V_{t}\left[\frac{F}{Q}\right]\right\}$, with $Q$ varying depending on the temporal resolution and length of the sequence. In all our experiments reported below, we adjusted $Q$ so that $\frac{F}{Q}=32$, replacing $V_{t}[1]$, which is not very informative, with the pure DC component $\mathcal{S}[1]$. 


\section{ANALYSIS}

One way to use these $V_{l}$ descriptors could be use them as measures of similarity between locations and perform a regionbased segmentation. However, here we wish to identify the areas of motion first.

\subsection{Dimension reduction}

To this end, we first reduce the dimensionality of the data via a Principal Component Analysis [12]. This is justified because there is some redundancy in the descriptors due to the recursive merging process used to compute them. The decriptors were whitened (normalized to zero-mean and unit variance). PCA was performed with scikit-learn [13]. Experiments showed that a dimensionality reduction from $E$ to 2 or 3 dimensions was sufficient. We broadly followed the heuristic that $K$ dimensions or less were sufficient for up to $2^{K}$ clusters

\subsection{Clustering}

Clustering was performed using k-means [14]. We again used the scikit-learn implementation. We typically chose 3-8 classes depending on the problem at hand. As shown on Fig. 3(c), the combination of dimension reduction and clustering is effective at labeling areas of motion correctly.

As a first illustration, we used this methodology to identified areas of motion in the tail of a fish embryo to detect blood vessels (see Fig. 3). Image sequence was acquired at 100 frames per second in a bright field inverted microscope at $40 \times$ magnification.

For a second illustration, we analyze a sequence of exvivo human ciliated cell, part of a nasal biopsy. The sequence was acquired at 358 frames per second in an inverted bright field microscope at $1000 \times$ magnification. The cilia at the surface of the cell are at the limit of resolution $(0.25-0.15 \mu \mathrm{m}$ in diameter, about $10 \mu \mathrm{m}$ in length). As shown on Fig. 4, our method can automatically distinguish between cilia beating patterns, which is an improvement on previous work [6].

\section{APPLICATION AND VALIDATION}

To demonstrate the potential of our method for the automated analysis of more complex motions, we propose to estimate the heart rate of fish embryos by measuring the change of velocity in the their tail artery over time. Even though it would appear at first glance that this motion is no longer stationary, we can still deal with it by performing our descriptor calculation on a squared temporal gradient, representing the square of the velocity, i.e. the kinetic energy.

I.e. instead of $I$ in Eq (1), we use $\nabla_{t}^{2}(I)=(I(i, j, t)-$ $I(i, j, t+1))^{2}$. With this, the temporal velocity variation (and

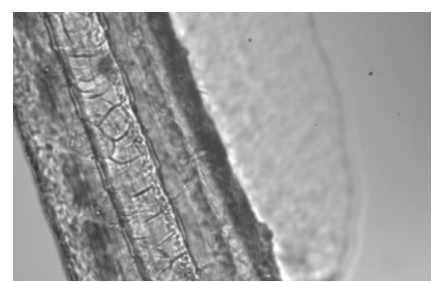

(a)

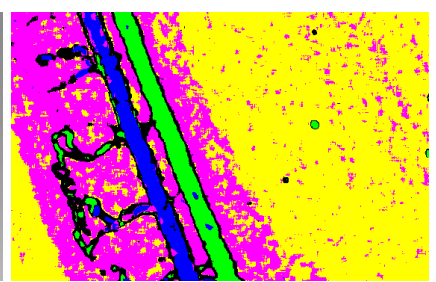

(b)

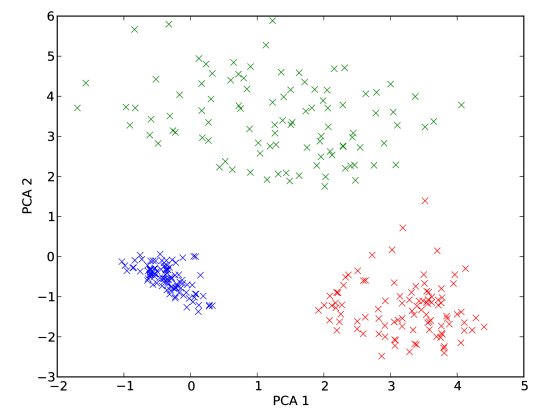

(c)
Fig. 3. Areas of motion identification in the tail of a fish embryo. (a) original image (c) PCA + k-means clustering ; (b) areas identification. The blue region is an artery and the green area a vein. The tail of the fish embryo also moves slightly and is identified as such (red area). The yellow area is the immobile background.

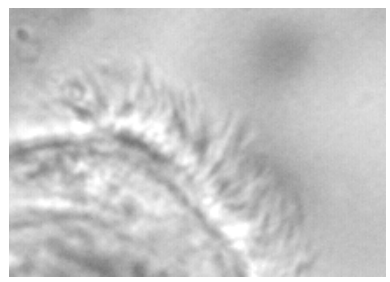

(a)

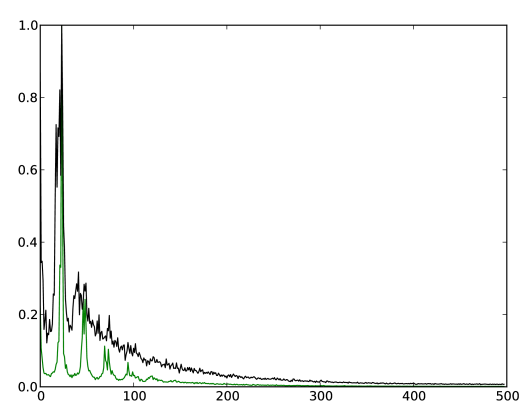

(c)

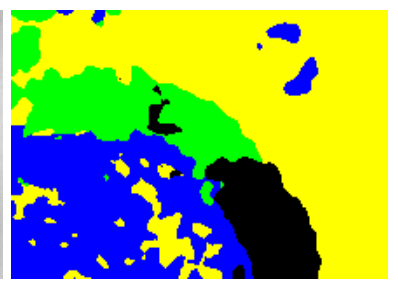

(b)
Fig. 4. Areas of motion identification on the surface of a ciliated cell. (a) original image; (b) areas identification and (c) corresponding FT in green and black areas. The green and black areas are associated with cilia beating in a different pattern, as illustrated in the average FT of their respective areas. 


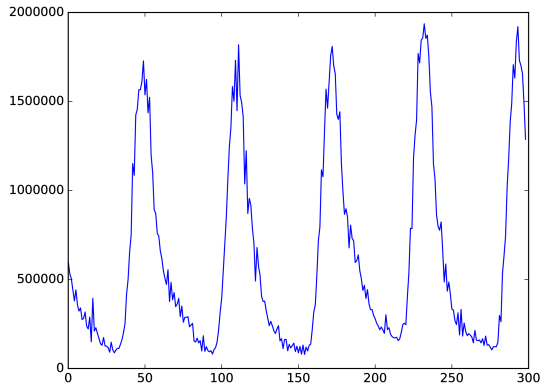

Fig. 5. Variation of the temporal squared gradient over time. A nearly periodic process is evident.

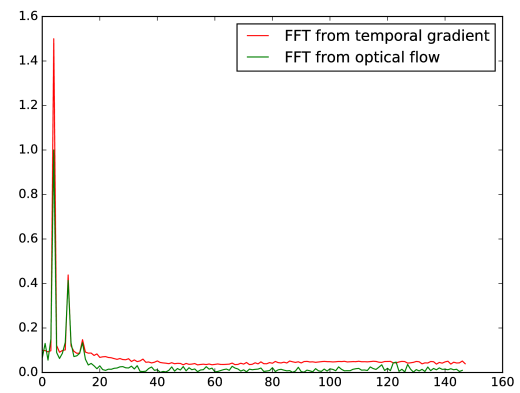

Fig. 6. Fourier analysis of the squared temporal gradient in a fish embry artery (in red) vs. that of the optical flow variations in the same area or motion (in green). The peak frequencies are almost exactly the same.

hence the kinetic energy variation) becomes a stationary process again, since the pattern of beating heart and hence of velocity change is periodic. This is illustrated in Fig. 5.

On Fig. 6, we show the result of the Fourier analysis of the variation of the optical flow magnitude averaged in the embryo tail artery vs. the same analysis on the squared temporal gradient. The peaks in both analyses are exactly the same, which suggests that the latter can be used to study the embryo's heart rythm.

To validate this observation, we perform this frequency analysis on a series of 15 sequences of blood flow in the tail of fish embryos. The ground truth consists of expert assessment of the embryo heart rhythms [8]. Results are shown on Fig. 7. As can be seen on this figure, the two analyses yield almost identical results over a large range of values (from 0.7 to 2.5 $\mathrm{Hz}$ ).

The main benefit of the approach that we propose in this article, is that it can be entirely automated with a high degree of robustness. A single measure (the squared temporal gradient) can be used for the areas-of-motion clustering proposed in section 3, identification of the area with the highest average motion (corresponding to the artery), and velocity analysis,

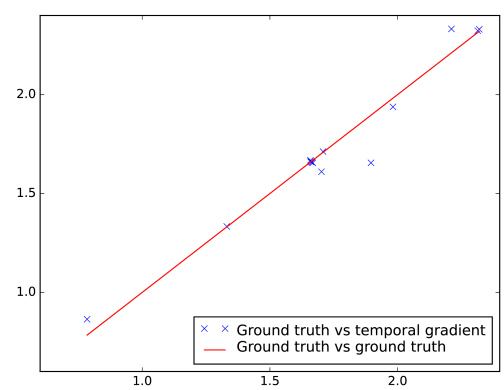

Fig. 7. Validation by comparing the squared temporal gradient Fourier analysis to the ground truth. The correlation is excellent overall over a large range of variations. The numerical values are in $\mathrm{Hz}$.

yielding the heart rate.

\section{CONCLUSION}

In this article, we have proposed a methodology for identifying and analyzing motion in sequences where the motion is spatially variant but stationary in time: in a given area, the apparent motion remains unchanged but its characteristics vary with the location in the image. We call this methodology "area-of-motion characterization". We carry it out by proposing descriptors corresponding to a multi-scale analysis of temporal Fourier coefficients. The characterization is performed by PCA for dimensionality reduction followed by k-means clustering. We have shown that our proposed methodology works well on various applications, in particular cilia beating and blood flow analyses. In the latter case, we have validated our proposed methodology by comparing our estimation of the frequency of the blood flow variations against the heart rhythm, with very good performance. The advantages of our proposed methodology are its versatility, simplicity, and the ability to perform joint area identification and analysis. The ability of the proposed method to distinguish between subtle motion patterns will be exploited in future work.

\section{Thanks}

The fish embryo were provided by Marc Léonard, Emilie Barbeau and Naima El Khallouki at L'Oréal Research and Innovation, Aulnay sous Bois, France. The nasal biopsy sequences were provided by Bruno Louis, Emilie Béquignon and André Coste at Institut Mondor de Recherche Biomédicale, Université Paris-Est, France. 


\section{REFERENCES}

[1] Richard A Robb, Biomedical imaging, visualization, and analysis, John Wiley \& Sons, Inc., 1999.

[2] Michael M Chang, A Murat Tekalp, and M Ibrahim Sezan, "Simultaneous motion estimation and segmentation," IEEE transactions on image processing, vol. 6, no. 9, pp. 1326-1333, 1997.

[3] Ross Cutler and Larry S. Davis, "Robust real-time periodic motion detection, analysis, and applications," IEEE Transactions on Pattern Analysis and Machine Intelligence, vol. 22, no. 8, pp. 781-796, 2000.

[4] A-R Mansouri and Janusz Konrad, "Multiple motion segmentation with level sets," IEEE Transactions on Image Processing, vol. 12, no. 2, pp. 201-220, 2003.

[5] Fabien Lauer and Christoph Schnörr, "Spectral clustering of linear subspaces for motion segmentation," in 2009 IEEE 12th International Conference on Computer Vision. IEEE, 2009, pp. 678-685.

[6] E. Puybareau, H. Talbot, G. Pelle, B. Louis, J.-F. Papon, A. Coste, and L. Najman, "A regionalized automated measurement of ciliary beating frequency," in Biomedical Imaging (ISBI), IEEE 12th International Symposium on. IEEE, 2015, pp. 528-531.

[7] E Puybareau, Hugues Talbot, E Bequignon, B Louis, G Pelle, J-F Papon, A Coste, and Laurent Najman, "Automating the measurement of physiological parameters: a case study in the image analysis of cilia motion," in 2016 IEEE International Conference on Image Processing (ICIP). IEEE, 2016, pp. 1240-1244.

[8] E. Puybareau, H. Talbot, and M. Leonard, "Automated heart rate estimation in fish embryo," in Image Processing Theory, Tools and Applications (IPTA), International Conference on, Orleans, November 2015, pp. 379-384.

[9] Gunnar Farnebäck, "Two-frame motion estimation based on polynomial expansion," in Scandinavian conference on Image analysis. Springer, 2003, pp. 363-370.

[10] Dennis Gabor, "Theory of communication. part 1: The analysis of information," Electrical Engineers-Part III: Radio and Communication Engineering, Journal of the Institution of, vol. 93, no. 26, pp. 429-441, 1946.

[11] Pierre Goupillaud, Alex Grossmann, and Jean Morlet, "Cycle-octave and related transforms in seismic signal analysis," Geoexploration, vol. 23, no. 1, pp. 85-102, 1984.
[12] Svante Wold, Kim Esbensen, and Paul Geladi, "Principal component analysis," Chemometrics and intelligent laboratory systems, vol. 2, no. 1-3, pp. 37-52, 1987.

[13] Fabian Pedregosa, Gaël Varoquaux, Alexandre Gramfort, Vincent Michel, Bertrand Thirion, Olivier Grisel, Mathieu Blondel, Peter Prettenhofer, Ron Weiss, Vincent Dubourg, et al., "Scikit-learn: Machine learning in python," Journal of Machine Learning Research, vol. 12, no. Oct, pp. 2825-2830, 2011.

[14] Hugo Steinhaus, "Sur la division des corp materiels en parties," Bull. Acad. Polon. Sci, vol. 1, no. 804, pp. 801, 1956. 